\title{
Improved constraints on the coupling constants of axion-like particles to nucleons from recent Casimir-less experiment
}

\author{
G. L. Klimchitskaya ${ }^{1,2}$, V. M. Mostepanenko ${ }^{1,2, a}$ \\ ${ }^{1}$ Central Astronomical Observatory at Pulkovo of the Russian Academy of Sciences, St. Petersburg 196140, Russia \\ 2 Institute of Physics, Nanotechnology and Telecommunications, St. Petersburg State Polytechnical University, St. Petersburg 195251, Russia
}

Received: 18 March 2015 / Accepted: 8 April 2015 / Published online: 24 April 2015

(C) The Author(s) 2015. This article is published with open access at Springerlink.com

\begin{abstract}
We obtain improved constraints on the coupling constants of axion-like particles to nucleons from a recently performed Casimir-less experiment. For this purpose, the differential force between a Au-coated sphere and either the Au or the Si sector of a rotating disc, arising due to two-axion exchange, is calculated. Over a wide region of axion masses, from $1.7 \times 10^{-3} \mathrm{eV}$ to $0.9 \mathrm{eV}$, the obtained constraints are up to a factor of 60 stronger than the previously known ones following from the Cavendish-type experiment and measurements of the effective Casimir pressure.
\end{abstract}

\section{Introduction}

It is common knowledge that the proper QCD axions are pseudo-scalar particles which appear as a consequence of breaking the Peccei-Quinn symmetry [1], proposed to resolve the problem of strong $C P$ violation in $\mathrm{QCD}$. After the prediction of axions [2,3], a lot of experimental and theoretical work has been done on their search and investigation of their role in elementary particle physics, astrophysics, and cosmology [4-12]. At the moment, the originally introduced QCD axions, which are pseudo-Nambu-Goldstone bosons, are constrained to a very narrow band in parameter space [11], and many types of so-called axion-like particles are proposed in different models (see, for instance, the hadronic axions $[13,14]$ and the GUT axions $[15,16])$.

Axion-like particles interact with photons, electrons, and nucleons. Many searches of these particles are based on the use of helioscopes and haloscopes [17]. The helioscopes are created for the registration of axion-like particles generated in the sun [18-21]. The haloscopes exploit the idea that axionlike particles are possible constituents of dark matter [4,22] and fill all the space around us. Then, their coupling to photons can be detected using a cryogenic microwave cavity in

\footnotetext{
a e-mail: vmostepa@gmail.com
}

a strong magnetic field [11,23,24]. Many constraints on the parameters of axion-like particles were obtained also from different astrophysical processes (see, for instance, [25-32]). Thus, from the neutrino data of supernova SN 1987A the coupling constant of the hadronic axions to nucleons was shown to be less than $10^{-10}$ or larger than $10^{-3}$ with a narrow allowed region in the vicinity of $10^{-6}$ [26]. Stellar cooling by the emission of hadronic axions leads to the conclusion that for hadronic axions this interaction constant is less than $3 \times 10^{-10}[27,32]$. It is noted [27], however, that the emission rate suffers from significant uncertainties related to dense nuclear matter effects. A new strong limit on the axion mass and, thus, on the axion-to-nucleon interaction constant (which are connected for hadronic axions) was obtained from direct Chandra observations of the surface temperature of the isolated neutron star in Cassiopeia A and its cooling scenario [33].

The model-independent laboratory constraints on the coupling constants of axion-like particles with nucleons were obtained from neutron physics [34,35], Eötvos- and Cavendish-type experiments [36-38], and from measurements of the Casimir and Casimir-Polder force [39-42]. These constraints cover a wide range of masses of axion-like particles from $10^{-10} \mathrm{eV}$ to $20 \mathrm{eV}$. As was shown in [39-42] (see also [43] for a review), measurements of the Casimir interaction lead to stronger constraints on the coupling constants of axions to nucleons than those obtained from the Cavendish-type experiments. This corresponds to separation distances between the test bodies where the Casimir interaction becomes stronger than the gravitational one.

In this paper, we obtain improved constraints on the coupling constants of axion-like particles to the proton and the neutron following from the recently performed Casimir-less experiment [44]. This is a differential force measurement between a Au-coated sphere and either a $\mathrm{Au}$ sector or a $\mathrm{Si}$ sector of the structured disc deposited on a Si substrate and covered by the overlayers of $\mathrm{Cr}$ and $\mathrm{Au}$. In such a manner, 
the contribution of the Casimir force to the differential signal is subtracted, and the measurement result is determined solely by the difference in the forces due to the exchange of some hypothetical particles. By achieving the unprecedented force sensitivity of approximately $10^{-16} \mathrm{~N}$, an improvement of the constraints on the Yukawa-type corrections to Newtonian gravitation by a factor of $10^{3}$ was achieved [44]. The corrections of Yukawa type arise due to exchange of one scalar boson between two atoms of the laboratory test bodies [45] or from compact extra dimensions with a low-energy compactification scale [46]. Here, we use the same experimental results to improve the previously known laboratory constraints on the coupling constants of axion-like particles to nucleons. Taking into account that the test bodies are unpolarized [44] and the axion-like particles are pseudo-scalar, the additional axionic interaction arises due to two-axion exchange between the nucleons of the test bodies. Here, we strengthen the axion-to-nucleon coupling constants up to a factor of 60 within the wide region of axion masses from $1.7 \times 10^{-3}$ to $0.9 \mathrm{eV}$. All equations are written in the system of units with $\hbar=c=1$.

\section{Differential force between a sphere and a structured disc due to two-axion exchange}

In the experiment [44], a Au-coated sapphire sphere of $R=149.3 \mu \mathrm{m}$ radius interacts in vacuum with either a $\mathrm{Au}$ sector or a $\mathrm{Si}$ sector of the structured rotating disc of thickness $D=2.1 \mu \mathrm{m}$, and the difference in these interaction forces is an immediately measured quantity. The structured disc was deposited on a Si substrate and covered by the overlayers of $\mathrm{Cr}$ and $\mathrm{Au}$ of thicknesses $d_{\mathrm{Cr}}=10 \mathrm{~nm}$ and $d_{\mathrm{Au}}=150 \mathrm{~nm}$, respectively. Note that in [44] the concentric alternating strips of $\mathrm{Au}$ and $\mathrm{Si}$ have been used rather than a sectoral structure. This, however, does not influence our calculation of the differential force. An important point is that the thick overlayer of Au results in equal Casimir forces when the sphere bottom is above a Au sector or a Si sector. Thus, the Casimir force does not contribute to the measured differential force. The latter is determined by possible hypothetical interactions, such as the Yukawa-type correction to Newton's gravitational law [44] or the two-axion exchange between nucleons of the sphere and the structured disc under consideration here (if both these attractive interactions exist in nature and contribute to the measured differential force, the constraints imposed on each of them by the measurement data would be even stronger than those obtained in [44] and in this paper).

In this section, we consider the homogeneous Au sphere interacting due to two-axion exchange between nucleons with the structured Au/Si disc. We assume the pseudo-scalar character of the axion-nucleon interaction, which is appli- cable to wide classes of axion-like particles, specifically, to all GUT axions [47] with no connection between their mass and their interaction constant. Note that the account of the scalar coupling of the axions to fermions [47] or the interaction of the axions with electrons could only slightly increase the magnitude of the differential axionic force and, thus, only slightly strengthen the obtained constraints (see $[35,48]$ for the constraints on the scalar interaction of axions with nucleons).

We assume that the coordinate plane $(x, y)$ coincides with the upper plane of the disc and the $z$ axis is perpendicular to it. The origin of the coordinate system is chosen below the bottom point of the sphere nearest to the disc. Without loss of accuracy one can neglect by the finite size effects and consider the disc of infinitely large area [41,49]. The separation distance between the sphere and the disk is $a$, so that the sphere center is at $z=a+R$. The effective potential due to two-axion exchange between two nucleons situated at the points $\boldsymbol{r}_{1}$ of the sphere and $\boldsymbol{r}_{2}$ of the disc is given by $[36,50,51]$

$V_{k l}\left(\left|\boldsymbol{r}_{1}-\boldsymbol{r}_{2}\right|\right)=-\frac{g_{a k}^{2} g_{a l}^{2} m_{a}}{32 \pi^{3} m^{2}} \frac{K_{1}\left(2 m_{a}\left|\boldsymbol{r}_{1}-\boldsymbol{r}_{2}\right|\right)}{\left(\boldsymbol{r}_{1}-\boldsymbol{r}_{2}\right)^{2}}$

Here, $g_{a k}$ and $g_{a l}$ are the coupling constants of the interaction between an axion-like particle of mass $m_{a}$ and a proton $(k, l=p)$ or a neutron $(k, l=n)$, the mean mass of a nucleon is $m=\left(m_{n}+m_{p}\right) / 2$, and $K_{1}(z)$ is the modified Bessel function of the second kind. Equation (1) is applicable under the condition $\left|\boldsymbol{r}_{1}-\boldsymbol{r}_{2}\right| \gg 1 / m$, which is satisfied with a large safety margin because in the experiment $a>200 \mathrm{~nm}$ [44].

The additional force due to two-axion exchange, acting between a homogeneous sphere $(s)$ and a homogeneous disc $(d)$, was found in [40] by the summation of microscopic forces determined by the potential (1):

$$
\begin{aligned}
F_{\text {add }}(a)= & \frac{\pi m_{a}}{m^{2} m_{H}^{2}} C_{d} C_{s} \int_{a}^{2 R+a} \mathrm{~d} z_{1}\left[R^{2}-\left(z_{1}-R-a\right)^{2}\right] \\
& \times I\left(m_{a}, D, z_{1}\right),
\end{aligned}
$$

where

$$
\begin{aligned}
I\left(m_{a}, D, z_{1}\right) \equiv & \frac{\partial}{\partial z_{1}} \int_{-D}^{0} \mathrm{~d} z_{2} \int_{0}^{\infty} \rho \mathrm{d} \rho \\
& \times \frac{K_{1}\left(2 m_{a} \sqrt{\rho^{2}+\left(z_{1}-z_{2}\right)^{2}}\right)}{\rho^{2}+\left(z_{1}-z_{2}\right)^{2}} .
\end{aligned}
$$

Here, the coefficient $C_{d, s}$ for the disc and sphere materials is defined as

$C_{d, s}=\rho_{d, s}\left(\frac{g_{a p}^{2}}{4 \pi} \frac{Z_{d, s}}{\mu_{d, s}}+\frac{g_{a n}^{2}}{4 \pi} \frac{N_{d, s}}{\mu_{d, s}}\right)$,

where $\rho_{d, s}$ is the disc and sphere densities, and $Z_{d, s}$ and $N_{d, s}$ are the number of protons and the mean number of neutrons 
in the atoms of a disc and a sphere. The quantities $\mu_{d, s}$ are defined as $\mu_{d, s}=m_{d, s} / m_{H}$ where $m_{d, s}$ are the mean masses of the disc and sphere atoms, and $m_{H}$ is the mass of an atomic hydrogen, respectively. The values of $Z / \mu$ and $N / \mu$ for many elements with account of their isotopic composition can be found in [45].

The quantity $I$ defined in (3) can be equivalently represented in the form [40]

$$
\begin{aligned}
I\left(m_{a}, D, z_{1}\right)= & -\int_{1}^{\infty} \mathrm{d} u \frac{\sqrt{u^{2}-1}}{u} \mathrm{e}^{-2 m_{a} u z_{1}} \\
& \times\left(1-\mathrm{e}^{-2 m_{a} u D}\right) .
\end{aligned}
$$

Using (2) and (5), the magnitude of the differential additional force arising from the alternate interaction of the sphere with the $\mathrm{Au}$ and $\mathrm{Si}$ sectors of the structured disc is equal to

$$
\begin{aligned}
\left|\Delta F_{\text {add }}(a)\right|= & \frac{\pi m_{a}}{m^{2} m_{H}^{2}} C_{S}\left(C_{\mathrm{Au}}-C_{\mathrm{Si}}\right) \\
& \times \int_{1}^{\infty} \mathrm{d} u \frac{\sqrt{u^{2}-1}}{u}\left(1-\mathrm{e}^{-2 m_{a} u D}\right) \\
& \times \int_{a}^{2 R+a} \mathrm{~d} z_{1} \mathrm{e}^{-2 m_{a} u z_{1}}\left[R^{2}-\left(z_{1}-R-a\right)^{2}\right] .
\end{aligned}
$$

Here, $C_{\mathrm{Au}}$ and $C_{\mathrm{Si}}$ are defined by (4) for the $\mathrm{Au}$ and $\mathrm{Si}$ disc materials, respectively.

It is convenient to introduce the new integration variable $t=z_{1}-a$ in the integral with respect to $z_{1}$. After the integration with respect to $t$ is performed, the differential force (6) is given by

$$
\begin{aligned}
& \left|\Delta F_{\text {add }}(a)\right|=\frac{\pi}{2 m_{a} m^{2} m_{H}^{2}} C_{S}\left(C_{\mathrm{Au}}-C_{\mathrm{Si}}\right) \\
& \quad \times \int_{1}^{\infty} \mathrm{d} u \frac{\sqrt{u^{2}-1}}{u^{3}} \mathrm{e}^{-2 m_{a} u a}\left(1-\mathrm{e}^{-2 m_{a} u D}\right) \Phi\left(R, m_{a} u\right),
\end{aligned}
$$

where

$\Phi(r, z)=r-\frac{1}{2 z}+\mathrm{e}^{-2 r z}\left(r+\frac{1}{2 z}\right)$.

Note that Eq. (7) is the exact one. The differential force due to two-axion exchange can be obtained also using the proximity force approximation [52], where the spherical surface is replaced with infinitesimally small plane plates parallel to the disc. This again results in (7), but with $\Phi=R$. Such an approximate expression is only applicable under a condition $R \gg m_{a}^{-1}$.

In the next section, we apply Eq. (7) to a calculation of the differential force in the configuration of experiment [44] and obtain constraints on the coupling constants of axion-like particles to nucleons.

\section{Improved constraints on the coupling constants of axions to nucleons}

Now we take into account that in the experiment [44] the sphere was not homogeneous. It was made of sapphire $\left(\mathrm{Al}_{2} \mathrm{O}_{3}\right)$ and covered with the layers of $\mathrm{Cr}$ and $\mathrm{Au}$ of thicknesses $\Delta_{\mathrm{Cr}}=10 \mathrm{~nm}$ and $\Delta_{\mathrm{Au}}=250 \mathrm{~nm}$, respectively. Thus, the differential force between the sapphire core and the structured disc can be calculated by (7) with $C_{s}=C_{\mathrm{Al}_{2} \mathrm{O}_{3}}$ where $R$ is replaced with $R-\Delta_{\mathrm{Au}}-\Delta_{\mathrm{Cr}}$. Then one should add to the obtained result the differential forces between the structured plate and each of two spherical envelopes of external radia $R-\Delta_{\mathrm{Au}}$ and $R$ of thicknesses $\Delta_{\mathrm{Cr}}$ and $\Delta_{\mathrm{Au}}$, made of $\mathrm{Cr}$ and $\mathrm{Au}$, respectively. These differential forces are calculated similarly using (7). For instance, the differential force due to a Au envelope is found by subtracting from (7) with $C_{s}=C_{\mathrm{Au}}$ the differential force due to a Au sphere of radius $R-\Delta_{\mathrm{Au}}$ placed at a separation $a+\Delta_{\mathrm{Au}}$ from the structured plate:

$$
\begin{aligned}
& \left|\Delta F_{\mathrm{add}}^{\mathrm{Au}}(a)\right|=\frac{\pi}{2 m_{a} m^{2} m_{H}^{2}} C_{\mathrm{Au}}\left(C_{\mathrm{Au}}-C_{\mathrm{Si}}\right) \\
& \quad \times \int_{1}^{\infty} \mathrm{d} u \frac{\sqrt{u^{2}-1}}{u^{3}} \mathrm{e}^{-2 m_{a} u a}\left(1-\mathrm{e}^{-2 m_{a} u D}\right) \\
& \quad \times\left[\Phi\left(R, m_{a} u\right)-\mathrm{e}^{-2 m_{a} u \Delta_{\mathrm{Au}}} \Phi\left(R-\Delta_{\mathrm{Au}}, m_{a} u\right)\right] .
\end{aligned}
$$

By adding up the differential forces from the sapphire core and the two spherical layers, we arrive at the following result valid in the experimental configuration [44]:

$$
\begin{aligned}
& \left|\Delta F_{\text {add }}^{\exp }(a)\right|=\frac{\pi}{2 m_{a} m^{2} m_{H}^{2}}\left(C_{\mathrm{Au}}-C_{\mathrm{Si}}\right) \\
& \quad \times \int_{1}^{\infty} \mathrm{d} u \frac{\sqrt{u^{2}-1}}{u^{3}} \mathrm{e}^{-2 m_{a} u a}\left(1-\mathrm{e}^{-2 m_{a} u D}\right) X\left(m_{a} u\right),
\end{aligned}
$$

where

$$
\begin{aligned}
& X(z) \equiv C_{\mathrm{Au}}\left[\Phi(R, z)-\mathrm{e}^{-2 z \Delta_{\mathrm{Au}}} \Phi\left(R-\Delta_{\mathrm{Au}}, z\right)\right] \\
& +C_{\mathrm{Cr}} \mathrm{e}^{-2 z \Delta_{\mathrm{Au}}}\left[\Phi\left(R-\Delta_{\mathrm{Au}}, z\right)\right. \\
& \left.-\mathrm{e}^{-2 z \Delta_{\mathrm{Cr}}} \Phi\left(R-\Delta_{\mathrm{Au}}-\Delta_{\mathrm{Cr}}, z\right)\right] \\
& +C_{\mathrm{Al}_{2} \mathrm{O}_{3}} \mathrm{e}^{-2 z\left(\Delta_{\mathrm{Au}}+\Delta_{\mathrm{Cr}}\right)} \Phi\left(R-\Delta_{\mathrm{Au}}-\Delta_{\mathrm{Cr}}, z\right) .
\end{aligned}
$$

Note that the quantities $C$ for $\mathrm{Au}, \mathrm{Cr}$, and $\mathrm{Al}_{2} \mathrm{O}_{3}$ are defined by (4). The values of $Z / \mu$ and $N / \mu$ for atoms of $\mathrm{Au}, \mathrm{Cr}$, and $\mathrm{Si}$, and for a molecule of $\mathrm{Al}_{2} \mathrm{O}_{3}$, as well as $\rho$ for these materials, are presented in Table I of [41].

Now we are in a position to obtain constraints on the parameters of axion-like particles following from the measurement results of experiment [44]. Recall that neither the Si substrate under the structured disc nor the Au and Cr overlayers contribute to the measured differential force which is 


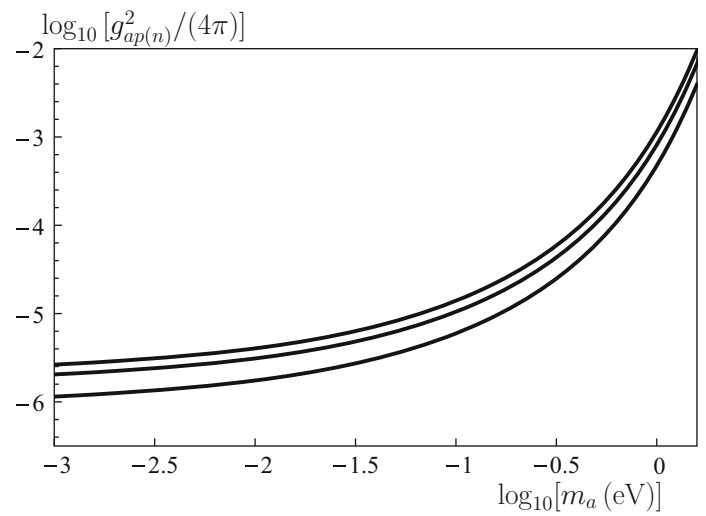

Fig. 1 Constraints on the coupling constants of axion-like particles to a proton or a neutron obtained from the Casimir-less experiment [44] are shown as functions of the axion mass. The lines from bottom to top are plotted under the conditions $g_{a p}^{2}=g_{a n}^{2}, g_{a n}^{2} \gg g_{a p}^{2}$, and $g_{a p}^{2} \gg g_{a n}^{2}$, respectively. The regions of the plane above each line are excluded and below each line are allowed

given by (10) and (11). However, when using the results of [44], it should be remembered that the experimental separation distances between the rotating disc and the sphere are equal to $z=a-d_{\mathrm{Au}}-d_{\mathrm{Cr}}=a-160 \mathrm{~nm}$.

In the experiment [44] no differential force was observed. This means that the quantity (10) arising due to two-axion exchange between the test bodies was less than the minimum detectable force,

$\Delta F_{\text {add }}^{\exp }(a) \leq \delta F(a)$.

According to Fig. 3 of [44], at separation distances $z=$ $200,400,700$, and $1000 \mathrm{~nm}$ (corresponding to $a=360,560$, 860 , and $1160 \mathrm{~nm}$ ) the minimum detectable force was equal to $\delta F=0.2,0.09,0.12$, and $0.12 \mathrm{fN}$, respectively. These values were determined at the $95 \%$ confidence level. We have found numerically the values of the axion-to-nucleon coupling constants $g_{a p}, g_{a n}$ and masses $m_{a}$ satisfying the inequality (12) with $\Delta F_{\text {add }}^{\exp }$ given by (10) and (11). The strongest constraints were obtained at $a=560 \mathrm{~nm}$.

The computational results for allowed and excluded values of the coupling constants $g_{a p(n)}^{2} /(4 \pi)$ as functions of the axion mass $m_{a}$ are presented in Fig. 1. The three lines from bottom to top are plotted under the conditions $g_{a p}^{2}=g_{a n}^{2}$, $g_{a n}^{2} \gg g_{a p}^{2}$, and $g_{a p}^{2} \gg g_{a n}^{2}$, respectively. The regions of the plane $\left(m_{a}, g_{a p(n)}^{2}\right)$ above each line are excluded by the experimental results, and the regions below each line are allowed. As can be seen in Fig. 1, with increasing $m_{a}$ the strength of the obtained constraints quickly decreases, and they become not competitive.

It is interesting to compare the constraints of Fig. 1 with previously obtained strongest laboratory constraints on axion-like particles. This comparison is presented in Fig. 2 under the most reasonable condition, $g_{a p}=g_{a n}$ [36]. In

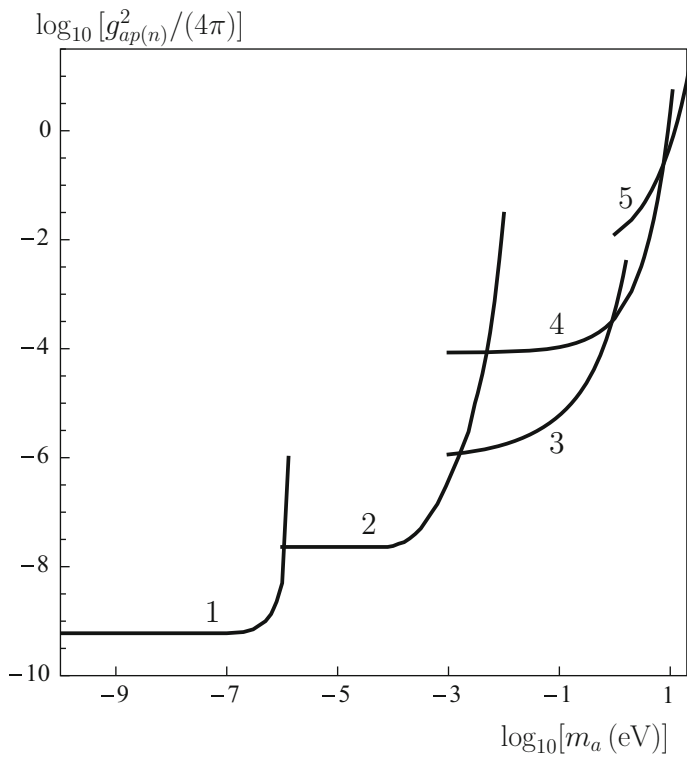

Fig. 2 Constraints on the coupling constants of axion-like particles to a proton and a neutron obtained under the condition $g_{a p}^{2}=g_{a n}^{2}$ from the magnetometer measurements [34] (line 1), from a Cavendish-type experiment $[37,38]$ (line 2), in this work from the Casimir-less experiment [44] (line 3), from measurements of the effective Casimir pressure $[41,55,56]$ (line 4), and from measurements of the lateral Casimir force between corrugated surfaces $[42,57,58]$ (line 5). The regions of the plane above each line are excluded and the regions below each line are allowed

Fig. 2, the line 1 shows the constraints found from the magnetometer measurements using spin-polarized $\mathrm{K}$ and ${ }^{3} \mathrm{He}$ atoms [34] (note also the recent limits on a product of the pseudo-scalar and scalar axion-to-nucleon interaction constants obtained $[53,54]$ from the magnetometer experiment with ${ }^{3} \mathrm{He}$ and ${ }^{129} \mathrm{Xe}$ atoms). The line 2 shows the constraints derived [38] from the Cavendish-type experiment [37]. The line 3 demonstrates the constraints obtained in this paper from the Casimir-less experiment [44]. This line reproduces the lowest line of Fig. 1. The constraints found [41] from measurements of the effective Casimir pressure by means of a micromachined oscillator $[55,56]$ are presented by the line 4. Finally, the line 5 shows the constraints derived [42] from measurements of the lateral Casimir force between sinusoidally corrugated surfaces $[57,58]$. The regions of the $\left(m_{a}, g_{a p(n)}^{2}\right)$ plane above all lines are excluded by the experimental results, and the regions below each line are allowed.

As can be seen in Fig. 2, the constraints of line 3 obtained in this paper are significantly improved in comparison with the previously known ones in the wide region of axion masses from $1.7 \times 10^{-3} \mathrm{eV}$ to $0.9 \mathrm{eV}$. In the region from $1.7 \times 10^{-3} \mathrm{eV}$ to $4.9 \times 10^{-3} \mathrm{eV}$ our constraints strengthen the constraints obtained [38] from a Cavendish-type experiment [37], whereas in the region from $4.9 \times 10^{-3} \mathrm{eV}$ to $0.9 \mathrm{eV}$ they are stronger than the constraints found [41] from measurements of the effective Casimir pressure $[55,56]$. The 
largest strengthening of previously known constraints by a factor of 60 holds for $m_{a}=4.9 \times 10^{-3} \mathrm{eV}$. Thus, the Casimirless experiment leads to stronger constraints not only on the Yukawa-type corrections to Newton's gravitational law [44], but on the coupling constants of axion-like particles to nucleons as well.

\section{Conclusions and discussion}

In the foregoing we have used the experimental results of recent Casimir-less experiment to derive the constraints on the axion-to-nucleon coupling constants. The obtained constraints strengthen the previously known ones following from a Cavendish-type experiment and from measurements of the effective Casimir pressure. The strengthening up to a factor of 60 is achieved over the wide region of axion masses from $1.7 \times 10^{-3} \mathrm{eV}$ to $0.9 \mathrm{eV}$.

It should be remarked that the results of the Casimir-less experiment used here to obtain the constraints on the axionnucleon interaction are unambiguous in the sense that they do not depend on any theory of the Casimir force. This is different, for instance, from the constraints obtained [41] from the measure of agreement between measured effective Casimir pressure and theory (see the line 4 in Fig. 2). It is well known that precise experiments on measuring the Casimir interaction between metallic surfaces agree with the extrapolation of a dielectric function to zero frequency using the plasma model, whereas a literally understood theory suggests to use the Drude model at low frequencies [52,55,56,59-62]. Recently the decisive experiment has been proposed [63] where the theoretical predictions of both approaches differ by a factor of order $10^{3}$. The first measurements performed in the framework of this proposal are in favor of the plasma model extrapolation [64]. However, a fundamental understanding of the physical mechanisms behind this problem is still missing. Because of this, the constraints obtained here, which are independent on the theory-experiment comparison for the Casimir forces, are of particular value.

In the past, both measurements of the Casimir interaction and the Casimir-less experiment were used for obtaining constraints on the Yukawa-type corrections to Newtonian gravity [44,52,55,56,65-70]. In Refs. [39-43] and in this paper it is shown that the same experiments also lead to competitive constraints on the coupling constants of the axion-to-nucleon interaction. It should be remembered, however, that the Yukawa potential arises due to the exchange of one scalar particle, whereas the spin-independent potential (1), used to constrain the parameters of axion-like particles, results from the exchange of two particles. The latter makes the obtained constraints relatively weak. Thus, in the future it seems promising to perform measurements of the Casimir interaction and Casimir-less experiment using polar- ized (magnetized) test bodies. In this way one could obtain much stronger constraints on the parameters of axion-like particles by exploiting a spin-dependent interaction potential arising due to one-particle exchange between the nucleons.

Acknowledgments The authors are grateful to R. S. Decca for useful information as regards his experiment.

Open Access This article is distributed under the terms of the Creative Commons Attribution 4.0 International License (http://creativecomm ons.org/licenses/by/4.0/), which permits unrestricted use, distribution, and reproduction in any medium, provided you give appropriate credit to the original author(s) and the source, provide a link to the Creative Commons license, and indicate if changes were made. Funded by $\mathrm{SCOAP}^{3}$.

\section{References}

1. R.D. Peccei, H.R. Quinn, Phys. Rev. Lett. 38, 1440 (1977)

2. S. Weinberg, Phys. Rev. Lett. 40, 223 (1978)

3. F. Wilczek, Phys. Rev. Lett. 40, 279 (1978)

4. J.E. Kim, Phys. Rep. 150, 1 (1987)

5. G.G. Raffelt, Stars as Laboratories for Fundamental Physics (University of Chicago Press, Chicago, 1996)

6. L.J. Rosenberg, K.A. van Bibber, Phys. Rep. 325, 1 (2000)

7. G.G. Raffelt, J. Phys. A: Math. Theor. 40, 6607 (2007)

8. J.E. Kim, G. Carosi, Rev. Mod. Phys. 82, 557 (2010)

9. J. Jaeckel, A. Ringwald, Annu. Rev. Nucl. Part. Sci. 60, 405 (2010)

10. M. Khlopov, Fundamentals of Cosmic Particle Physics (CISP/Springer, Cambridge, 2012)

11. K. Baker et al., Ann. Phys. (Berlin) 525, A93 (2013)

12. M. Kawasaki, K. Nakayama, Annu. Rev. Nucl. Part. Sci. 63, 69 (2013)

13. J.E. Kim, Phys. Rev. Lett. 43, 103 (1979)

14. M.A. Shifman, A.I. Vainstein, V.I. Zakharov, Nucl. Phys. B 166, $493(1980)$

15. A.P. Zhitnitskii, Sov. J. Nucl. Phys. 31, 260 (1980)

16. M. Dine, F. Fischler, M. Srednicki, Phys. Lett. B 104, 199 (1981)

17. P. Sikivie, Phys. Rev. Lett. 51, 1415 (1983)

18. A.V. Derbin, A.L. Frolov, L.A. Mitropol'sky, V.N. Muratova, D.A. Semenov, E.V. Unzhakov, Eur. Phys. J. C 62, 755 (2009)

19. A.V. Derbin, V.N. Muratova, D.A. Semenov, E.V. Unzhakov, Phys. Atom. Nucl. 74, 596 (2011)

20. A.V. Derbin, S.V. Bakhlanov, I.S. Dratchnev, A.S. Kayunov, V.N. Muratova, Eur. Phys. J. C 73, 2490 (2013)

21. M. Arik et al., Phys. Rev. Lett. 107, 261302 (2011)

22. Yu.N. Gnedin, Int. J. Mod. Phys. A 17, 4251 (2002)

23. S.J. Asztalos et al., Phys. Rev. Lett. 104, 041301 (2010)

24. J.K. Hoskins et al., Phys. Rev. D 84, 121302 (2011)

25. G.G. Raffelt, Phys. Rev. D 33, 897 (1986)

26. J. Engel, D. Seckel, A.C. Hayes, Phys. Rev. Lett. 65, 960 (1990)

27. W.C. Haxton, K.Y. Lee, Phys. Rev. Lett. 66, 2557 (1991)

28. H. Schlattl, A. Weiss, G. Raffelt, Astropart. Phys. 10, 353 (1999)

29. E. Massó, J. Redondo, Phys. Rev. Lett. 97, 151802 (2006)

30. J. Jaeckel, E. Massó, J. Redondo, A. Ringwald, F. Takahashi, Phys. Rev. D 75, 013004 (2007)

31. P. Brax, C. van de Bruck, A.-C. Davis, Phys. Rev. Lett. 99, 121103 (2007)

32. G. Raffelt, Phys. Rev. D 86, 015001 (2012)

33. L.B. Leinson, J. Cosmol. Astropart. Phys. 08, 031 (2014)

34. G. Vasilakis, J.M. Brown, T.R. Kornack, M.V. Romalis, Phys. Rev. Lett. 103, 261801 (2009)

35. V.V. Voronin, V.V. Fedorov, I.A. Kuznetsov, JETP Lett. 90, 5 (2009) 
36. E.G. Adelberger, E. Fischbach, D.E. Krause, R.D. Newman, Phys. Rev. D 68, 062002 (2003)

37. D.J. Kapner, T.S. Cook, E.G. Adelberger, J.H. Gundlach, B.R. Heckel, C.D. Hoyle, H.E. Swanson, Phys. Rev. Lett. 98, 021101 (2007)

38. E.G. Adelberger, B.R. Heckel, S. Hoedl, C.D. Hoyle, D.J. Kapner, A. Upadhye, Phys. Rev. Lett. 98, 131104 (2007)

39. V.B. Bezerra, G.L. Klimchitskaya, V.M. Mostepanenko, C. Romero, Phys. Rev. D 89, 035010 (2014)

40. V.B. Bezerra, G.L. Klimchitskaya, V.M. Mostepanenko, C. Romero, Phys. Rev. D 89, 075002 (2014)

41. V.B. Bezerra, G.L. Klimchitskaya, V.M. Mostepanenko, C. Romero, Eur. Phys. J. C 74, 2859 (2014)

42. V.B. Bezerra, G.L. Klimchitskaya, V.M. Mostepanenko, C. Romero, Phys. Rev. D 90, 055013 (2014)

43. G.L. Klimchitskaya, V.M. Mostepanenko, Gravit. Cosmol. 21, 1 (2015)

44. Y.-J. Chen, W.K. Tham, D.E. Krause, D. López, E. Fischbach, R.S. Decca, arXiv: $1410.7267 \mathrm{v} 1$

45. E. Fischbach, C.L. Talmadge, The Search for Non-Newtonian Gravity (Springer, New York, 1999)

46. I. Antoniadis, N. Arkani-Hamed, S. Dimopoulos, G. Dvali, Phys. Lett. B 436, 257 (1998)

47. J.E. Moody, F. Wilczek, Phys. Rev. D 30, 130 (1984)

48. S. Hoedl, F. Fleischer, E.G. Adelberger, B.R. Heckel, Phys. Rev. Lett. 106, 041801 (2011)

49. C.R. Jamell, R.S. Decca, Int. J. Mod. Phys. A 26, 3742 (2011)

50. S.D. Drell, K. Huang, Phys. Rev. 91, 1527 (1953)

51. F. Ferrer, M. Nowakowski, Phys. Rev. D 59, 075009 (1999)

52. M. Bordag, G.L. Klimchitskaya, U. Mohideen, V.M. Mostepanenko, Advances in the Casimir Effect (Oxford University Press, Oxford, 2009)

53. K. Tullney et al., Phys. Rev. Lett. 111, 100801 (2013)
54. Y.V. Stadnik, V.V. Flambaum, Eur. Phys. J. C 75, 110 (2015)

55. R.S. Decca, D. López, E. Fischbach, G.L. Klimchitskaya, D.E. Krause, V.M. Mostepanenko, Phys. Rev. D 75, 077101 (2007)

56. R.S. Decca, D. López, E. Fischbach, G.L. Klimchitskaya, D.E. Krause, V.M. Mostepanenko, Eur. Phys. J. C 51, 963 (2007)

57. H.-C. Chiu, G.L. Klimchitskaya, V.N. Marachevsky, V.M. Mostepanenko, U. Mohideen, Phys. Rev. B 80, 121402(R) (2009)

58. H.-C. Chiu, G.L. Klimchitskaya, V.N. Marachevsky, V.M. Mostepanenko, U. Mohideen, Phys. Rev. B 81, 115417 (2010)

59. G.L. Klimchitskaya, U. Mohideen, V.M. Mostepanenko, Rev. Mod. Phys. 81, 1827 (2009)

60. C.-C. Chang, A.A. Banishev, R. Castillo-Garza, G.L. Klimchitskaya, V.M. Mostepanenko, U. Mohideen, Phys. Rev. B 85, 165443 (2012)

61. A.A. Banishev, G.L. Klimchitskaya, V.M. Mostepanenko, U. Mohideen, Phys. Rev. Lett. 110, 137401 (2013)

62. A.A. Banishev, G.L. Klimchitskaya, V.M. Mostepanenko, U. Mohideen, Phys. Rev. B 88, 155410 (2013)

63. G. Bimonte, Phys. Rev. Lett. 112, 240401 (2014)

64. R.S. Decca, Bull. Am. Phys. Soc. 60, N1, M35.00003 (2015). http:// meeting.aps.org/Meeting/MAR15/Session/M35.3

65. R.S. Decca, D. López, E. Fischbach, D.E. Krause, C.R. Jamell, Phys. Rev. Lett. 94, 240401 (2005)

66. V.B. Bezerra, G.L. Klimchitskaya, V.M. Mostepanenko, C. Romero, Phys. Rev. D 81, 055003 (2010)

67. V.B. Bezerra, G.L. Klimchitskaya, V.M. Mostepanenko, C. Romero, Phys. Rev. D 83, 075004 (2011)

68. G.L. Klimchitskaya, U. Mohideen, V.M. Mostepanenko, Phys. Rev. D 86, 065025 (2012)

69. G.L. Klimchitskaya, U. Mohideen, V.M. Mostepanenko, Phys. Rev. D 87, 125031 (2013)

70. G.L. Klimchitskaya, V.M. Mostepanenko, Gravit. Cosmol. 20, 3 (2014) 